\title{
Metaphysics and mathematics: Perspectives on reality
}

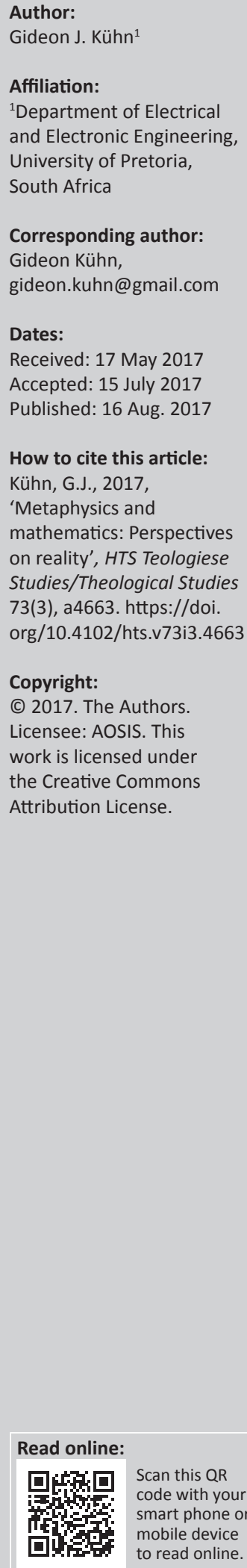

\begin{abstract}
The essence of number was regarded by the ancient Greeks as the root cause of the existence of the universe, but it was only towards the end of the 19th century that mathematicians initiated an in-depth study of the nature of numbers. The resulting unavoidable actuality of infinities in the number system led mathematicians to rigorously investigate the foundations of mathematics. The formalist approach to establish mathematical proof was found to be inconclusive: Gödel showed that there existed true propositions that could not be proved to be true within the natural number universe. This result weighed heavily on proposals in the mid20th century for digital models of the universe, inspired by the emergence of the programmable digital computer, giving rise to the branch of philosophy recognised as digital philosophy. In this article, the models of the universe presented by physicists, mathematicians and theoretical computer scientists are reviewed and their relation to the natural numbers is investigated. A quantum theory view that at the deepest level time and space may be discrete suggests a profound relation between natural numbers and reality of the cosmos. The conclusion is that our perception of reality may ultimately be traced to the ontology and epistemology of the natural numbers.
\end{abstract}

\section{Introduction}

When the 19th century mathematician George Cantor developed a set theory in his in-depth study of the natural numbers, a chain of reasoning was initiated that culminated in the incompleteness theorems of the mathematician Kurt Gödel and halting problem of the theoretical computer scientist Alan Turing. These theorems defined the boundaries of mathematical proofs. It applied to the digital models of the universe proposed by cosmologists and computer scientists such as Wolfram, Fredkin, Tegmark and others. In their views, the universe is fundamentally digital in nature, and reality may at the deepest level be a mathematical construct. We surmise that the ontology of digital models of the universe can be traced back to the ontology of the natural numbers through mathematical properties of the real numbers and other classes of numbers.

\section{Infinities in mathematics}

Towards the end of the 19th century, the German mathematician George Cantor (1845-1918) delved deeply into the ultimate properties of the natural numbers (the positive integers). To attain this objective, Cantor introduced the notion of sets (finite and infinite collections of elements), and developed the theory of relations between sets (Wikipedia 2017b:1 of 17). An important property of sets is a one-to-one relation between two sets. This relation exists if the members of the first set can be exhaustively paired with members of the second set without unpaired members remaining.

It is easy to realise that the ordinal numbers, that is, the sequence $1,2,3, \ldots{ }^{1}$ that we use when counting objects, can be extended by recursively adding 1 to the previous number up to any given number with no end in sight. The cardinality (size) of the set of natural numbers is infinite, larger than any number we can conceive. Cantor gave this infinity a name: he used the first letter of the Hebrew alphabet, calling the infinity aleph-0 (Stewart 1996).

The infinite set aleph-0 has some disturbing counter-intuitive properties (Gleiser 2014). Aleph-0 plus any finite number, no matter how large, is still aleph-0. Multiplying aleph-0 by any finite number again yields aleph-0. But the strangest property is that an infinite set can be placed into a one-to-one correspondence with a proper subset of itself. This contradicts the notion, stated by the Greek mathematician Euclid that 'The whole is greater than the part' (Wikipedia 2017a:2). As an example, consider the even numbers $2,4,6, \ldots$ which form a proper subset of the natural numbers

1.There is some disagreement amongst mathematicians on whether 0 should be included in the set of natural numbers or not. The natural numbers including 0 are referred to as whole numbers. The integers include negative numbers ...,-3, $-2,-1,0,1,2,3, \ldots$ 
because the subset does not contain the odd numbers 1, 3, 5, ... But we can count the even numbers up to infinity without missing any or counting a number twice. This is done by pairing each even number with its natural number counterpart, which is the even number divided by 2 . This property is very general and holds for any monotonically defined sequence of natural numbers.

Cantor studied the real numbers, which are numbers such as pi (the ratio of the circumference of a circle to its diameter) and the square root of 2 that require an infinite non-repeating decimal expansion for their numerical representation. He concluded that the real numbers were much more numerous than the natural numbers, and showed that it is impossible to count the real numbers or to effectively list the real numbers in any interval, no matter how small, on the number line. He designated the cardinality of the real numbers by the symbol aleph-1 and proved that aleph-1 is exponentially larger than aleph-0.

This notion of an 'infinity of infinities' brought him into conflict with many of his mathematical contemporaries, who did not accept his view of infinity. Some Christian theologians (particularly neo-Scholastics ${ }^{2}$ ) unfortunately misunderstood the mathematical notion of infinity: they saw Cantor's work as a challenge to the uniqueness of the absolute infinity in the nature of God (Dauben 1977:120; 1979:120, 143; Wikipedia 2017b:9 of 17). David Hilbert (18621943), the leading mathematician at the time, defended Cantor, declaring: 'From the paradise that Cantor with us unfolded, we hold our breath in awe; knowing we shall not be expelled' (Hilbert 1926).

\section{Formal logic}

In the first quarter of the 20th century, various antimonies arising from a study of what became known as naïve set theory were uncovered by mathematicians. The best known is probably Russell's paradox. He considered an unusual set: the set of all sets that are not members of themselves. He asked whether such a set is a member of itself. A valid line of logic reasoning brought a contradiction: if the set is a member of itself, then it is not a member of itself and if it is not a member of itself, then it is a member of itself. Such a conclusion is not allowed in mathematics: a statement is either TRUE or FALSE: there are no alternative 'truths'.

These antimonies led mathematicians to rigorously examine the foundations of mathematics. Formal logic with its emphasis on strict rules for induction, deduction and inference was perceived as a reliable means to this end. Various axiomatic systems such as Peano arithmetic, the Zermelo-Fraenkel system of set theory, as well as the system in Russels's Principia Mathematica, were believed by many to prove the soundness of the foundations of mathematics.

2.Neo-Scholasticism (also known as neo-scholastic Thomism or neo-Thomism because of the great influence of the writings of St. Thomas Aquinas on the movement) is a of the great influence of the writings of St. Thomas Aquinas on the movement) is a
revival and development of medieval scholasticism in Roman Catholic theology and revival and development of medieval scholasticism in Roman Catho
philosophy which began in the second half of the 19th century.
The leading mathematician in the early 20th century, David Hilbert, together with other mathematical logicians, initiated the discipline of metamathematics, which is a study of the laws of mathematics itself. An informal definition states that ' $2+2=4^{\prime}$ is a mathematical statement, while ' $2+2=4$ is valid' is a metamathematical statement.

The principal school in the search for a sound basis for mathematics was that of the formalist approach, and David Hilbert was the leading proponent, culminating in what is known as Hilbert's programme (Barrow 2000:276; Penrose 1989:129). He believed that mathematics could be formulated on a solid and complete logical foundation. In principle, this could be performed by showing that all of mathematics follows from a correctly chosen finite system of axioms; and that some such axiom system is provably consistent.

An axiom or postulate is a statement that is taken to be true to serve as a premise or starting point for further reasoning and argument, following the rules of logic. The oldest extant set of axioms is those formulated by Euclid in his Elements some 2300 years ago.

In the study of geometrical properties of lines, triangles and circles, Euclid postulated (Wikipedia 2017a: 2 of 15):

'To draw a straight line from any point to any point'.

'To produce [extend] a finite straight line continuously in a straight line'.

'To describe a circle with any centre and distance [radius]'.

'That all right angles are equal to one another'.

'That, if a straight line falling on two straight lines make the interior angles on the same side less than two right angles, the two straight lines, if produced indefinitely, meet on that side on which are the angles less than the two right angles'.

The convoluted expression for axiom 5 is generally known as the parallel postulate, which is informally interpreted that parallel lines never meet. For at least 1000 years, geometers were troubled by the disparate complexity of the fifth postulate compared to the first four, and suspected that it could be proved as a theorem from the first four.

Because the angles of a triangle sum to two right angles, we might argue that a triangle formed with two right angles results in the third angle being $0^{\circ}$, implying that the two lines emanating from the right angled side never meet. However, proof that the three angles of the triangle sum to two right angles depends on the truth of the parallel postulate. Thus, we fall into a circular reasoning trap, which proves nothing at all.

In the 19th century, Gauss, Bolyai, Lobachevsky and other mathematicians showed definitively that the parallel postulate is independent of the first four (Ball 1960:45; Wikipedia 2017a: 9 of 15). The parallel postulate is an assumption, a choice from alternative axioms. The newly developed non-Euclidean geometry allowed 'parallel' lines to intersect. As an example, longitudinal lines on the earth 
(a sphere) are geodesics ('straight' lines on a curved surface) that meet at the poles.

It is clear that defining a set of axioms satisfying Hilbert's requirements was a formidable task: the axioms must be independent, self-consistent and sufficiently diverse to cover all of mathematics. It came as a shock to Hilbert when Kurt Gödel (1906-1978), an Austrian, later American, logician, mathematician and philosopher published a paper in 1931 showing that such a set of axioms from which all theorems about the natural numbers can be proven did not exist (Gödel 1931). Gödel's first incompleteness theorem states that no consistent system of axioms relating to arithmetic is capable of proving all truths about the relations of the natural numbers. For any such system, there will always be statements about the natural numbers that are true, but that are unprovable within the system. The second incompleteness theorem, an extension of the first, showed that such a system cannot demonstrate its own consistency. Adding more axioms to the initial set to remove inconsistencies could not solve the problem. Working from inside the system did not allow theoreticians to transcend the limits inherent in a closed system.

\section{Computable numbers}

The British scientist and mathematician, Alan Turing (1912-1954), who is widely regarded as the father of theoretical computer science and artificial intelligence, developed a formalisation of the concepts of algorithm and computation. He proposed an abstract construction, now known as the Turing machine, which can be considered an idealised model of a digital computer able to simulate the workings of any digital computer. In 1936, Turing published a paper on a decision problem posed by Hilbert. In this article, Turing (1937; 1938) reformulated Gödel's (1931) results on the limits of proof and computation, replacing Gödel's universal arithmetic-based formal language with the formal and simple hypothetical devices that became known as Turing machines. He proved that his 'universal computing machine' would be capable of performing any conceivable mathematical computation if it were representable as an algorithm, which is an effective finite step-by-step procedure to compute a mathematical function. He went on to prove that there was no reliable solution to Hilbert's decision problem. He showed that it was not generally possible to determine beforehand whether a Turing machine working on a decision problem would stop calculating in a finite time and deliver a verdict 'TRUE' or 'FALSE'. This is now known as the halting problem.

Consider Goldbach's conjecture, which states that all even numbers greater than 4 can be expressed as the sum of two (odd) prime numbers, ${ }^{3}$ for example, $12=5+7$ (Wikipedia 2017d). Goldbach's conjecture was formulated 275 years ago. Despite substantial effort by leading mathematicians since then, the conjecture remains unproven. A computer-based search checking each even number up to the number 4 million trillion ( 4 followed by 18

3.A prime number is a natural number that is not divisible by a natural number greate than 1 and smaller than the number itself. zeros) yielded no counterexample. If the conjecture is false, a counterexample will exist and will eventually be found although it may take a time of cosmological dimension before the counterexample is reached. If the conjecture is true, the computer will continue forever without coming to a halt. It is not known whether the Goldbach's conjecture is true; thus, it is not possible to predict whether a computer working on the decision problem 'The Goldbach's conjecture is TRUE' will halt in a finite time. ${ }^{4}$

Surveying these momentous historical developments in mathematics, the present-day philosopher Roberto Unger invalidated Hilbert's ironic statement 'From the paradise that Cantor with us unfolded, we hold our breath in awe; knowing we shall not be expelled':

... mathematics before Gödel and Turing was on a track defined by Hilbert's ambition to reduce it to a closed system under axioms. Then Gödel and others appeared, and drove mathematics out of a paradise to which it has never since been able to return. (Unger \& Smolin 2015)

As a consequence, mathematics lost its claim that mathematical insight represents a shortcut to eternal truths about incorruptible objects. This result weighed heavily on philosophers of science in formulating new views on ontology and epistemology of science.

The Argentine-American computer scientist Gregory Chaitin (2007), who found a new proof of Gödel's incompleteness theorems, states that his own findings in mathematical logic and algorithmic information theory show that there are mathematical facts that are true for no reason, they are true by accident. They are random mathematical facts (Wikipedia 2017e:1 of 3). He proposes that mathematicians must abandon any hope of proving those mathematical facts and adopt a quasi-empirical methodology. As claims of consistency of any system are usually unprovable, many theorems in that system would remain a matter of belief or non-rigorous kinds of justifications.

\section{The universe as a computer}

The emergence of the programmable digital computer in the 1940s influenced many scientists in their search for understanding the cosmos.

A hypothesis that the universe is an enormous digital computer was first proposed by Konrad Zuse, a German computer pioneer, often regarded as the inventor of the modern computer, in 1941 (Wikipedia 2017f.:1, 5 of 9).

Others who have modelled the universe as a giant computer include Stephen Wolfram, a British-born American computer scientist, physicist and businessman. ${ }^{5}$ The concept of a cellular automaton, which is a discrete mathematical model

4.This statement does not exclude the possibility that in future, a gifted mathematician might find a proof of the conjecture through a new imaginative approach using known properties of the natural numbers.

5.Wolfram developed and marketed the commercially successful symbolic maths programme Mathematica. 
studied in computability theory, mathematics and many branches of science, played an important role in Wolfram's view of the universe.

\section{Cellular automata}

A cellular automaton consists of a regular grid of cells, each being in one of a finite number of states, such as, in the simplest case, two states designated by the terms 0 and 1 or $\mathrm{OFF}$ and $\mathrm{ON}$. Each cell is connected to a finite number of cells, called its neighbours. In a one-dimensional cellular automation, each cell has two neighbours, one on its left and one on its right, except at the edges, where there would only be one neighbour. Communication to any cell in the automation occurs only through information passed on to it by its neighbours. Conversely, communication from any cell to the other cells again only occurs through its neighbours.

The dynamics of a cellular automaton is determined by continuous updating of the states of the cells in discrete time increments. The update is mediated by a transition function, usually a mathematical formula that takes as input the state of a particular cell and the states of its neighbours and delivers an output that switches the chosen cell to a new state.

Wolfram published a controversial book with the title A New Kind of Science (Wolfram 2002), which presents an empirical study of very simple computational systems like cellular automata. Wolfram's conclusion was that the universe is digital in its nature, and runs on fundamental laws which can be described as simple programmes. He argues that these types of systems, rather than traditional mathematics, are needed to model and understand complexity in nature. He predicted that a realisation of this within the scientific community will have a major and revolutionary impact on physics, chemistry and biology and the majority of the scientific areas in general.

Critics questioned his assumption that simple systems such as cellular automata are sufficiently multifaceted to describe the degree of complexity present in evolved systems. Some viewed Wolfram's theory as a minor development and dispute Wolfram's claim of a paradigm shift. Others found that the work contained valuable insights and refreshing ideas, amongst them Chaitin, who have been mentioned earlier.

\section{Digital philosophy}

Digital philosophy is a direction in philosophy and cosmology advocated by certain mathematicians and theoretical physicists, including Edward Fredkin, Seth Lloyd and Jürgen Schmidhuber, all associated in one form or another with the Massachusetts Institute of Technology.

The school of digital philosophy, which is known as pancomputationalists, claims that all the physical processes of nature are forms of computation or information processing at the most fundamental level of reality. They believe that biology reduces to chemistry which, in turn, reduces to physics which, in turn, reduces to the computation of information. Fredkin's digital philosophy contains several fundamental ideas: everything in physics and physical reality must have a digital informational representation. All changes in physical nature are consequences of digital informational processes. Nature is finite and digital. Pancomputationalism is related to several larger schools of philosophy: atomism, determinism, mechanism, monism, naturalism, philosophical realism, reductionism and scientific empiricism.

Digital philosophy is considered to be a modern interpretation of Gottfried Leibniz's 17th century monist metaphysics as exposited in his Monadologie (Schrecker \& Schrecker 1965; Wikipedia 2017c:8 of 28). According to Leibniz, monads are the ultimate elements of the universe. The monads are 'substantial forms of being' with remarkable properties: they are eternal, indecomposable, individual, subject to their own laws, un-interacting, and each reflecting the entire universe in a pre-established harmony. Monads are centres of force; substance is force, while space, matter and motion are merely phenomenal. Digital philosophy appears to replace Leibniz's monads with aspects of the theory of cellular automata.

Lloyd (2006), a member of the MIT Research Laboratory of Electronics, contends that the universe itself is one big quantum computer ${ }^{6}$ producing what we see around us, and ourselves, as it runs a cosmic programme. According to Lloyd, once we understand the laws of physics completely, we will be able to use small-scale quantum computing to understand the universe completely as well.

\section{A mathematical universe}

A different view was presented by physicist and philosopher Max Erik Tegmark, a Swedish-American cosmologist who is a professor at the Massachusetts Institute of Technology and the scientific director of the Foundational Questions Institute.

Tegmark made the bold assertion that 'all structures that exist mathematically exist also physically' (Tegmark 2014:321 of 399). He has formulated the 'Ultimate Ensemble Theory of Everything', whose only postulate is that in structures that are complex enough to contain self-aware substructures, these substructures will subjectively perceive themselves as existing in a physically 'real' world. This idea is formalised as the Mathematical Universe Hypothesis (MUH), which implies that mathematical existence equals physical existence. Tegmark postulated a number of hypotheses to clarify his views.

The Computable Universe Hypothesis (CUH) states that the mathematical structure of our external physical reality is defined by computable functions that, by their definition, do

6.A quantum computer is a computing device that will exploit the quantum properties of atomic and subatomic particles to rapidly compute mathematical functions. The of atomic and subatomic particles to rapidly compute mathematical functions. The
technology is at present in an exploratory phase with realisation of a full-scale quantum computer somewhere in the future. 
not suffer the Gödel incompleteness properties. The Finite Universe Hypothesis (FUH) implies the $\mathrm{CUH}$ and eliminates all concerns about reality being undefined. The CUH or FUH may explain why our universe is so simple. Physics is so successfully described by mathematics because the physical world is completely mathematical; isomorphic to a mathematical structure and that we are simply uncovering it bit by bit.

The theory can be considered a form of Pythagoreanism or Platonism in that it posits the existence of mathematical entities; a form of mathematical monism in that it denies that anything exists except mathematical objects.

On responding to criticism of his theory, Tegmark offered the explanation that only Gödel-complete (fully decidable) mathematical structures have physical existence. This places a tight constraint on realisable universes, essentially placing an upper limit on complexity, but may have the attractive side effect of explaining the relative simplicity of our universe. The expressions for Newton's laws of motion and gravity, and the basic principles of Einstein's relativity theory and the theory of gravity, are in principle easy to understand, if not aesthetically pleasing, but the corresponding mathematical expressions tend to be complex.

Jürgen Schmidhuber, a computer scientist who works in the field of artificial intelligence, put forward a more restricted ensemble which admits only universe representations describable by constructive mathematics. The intuitionists, of whom the mathematician L.E.J. Bouwer (1881-1966) was the main exponent, stated that a mathematical object does not exist unless it can be constructed from whole numbers in a finite number of steps. In a modern view, this implies that the object only exists if it can be coded in a computer programme. He explicitly includes universe representations describable by halting programmes whose output bits converge after a finite time, but because of the undecidability of the halting problem, it may not be possible to predict the convergence time itself.

\section{Natural numbers}

Amongst the Pythagorean Sacred Discourses (2500 years ago), there is a dictum attributed to Arignote:

The eternal essence of number is the most providential cause of the whole heaven, earth and the region in between. Likewise it is the root of the continued existence of the gods and daimones, as well as that of divine men. (Wikipedia 2017i:3 of 16)

The German mathematician Leopold Kronecker (1823-1891) reportedly exclaimed 'God created the integers. All the rest is the work of Man' (Bell 1986). This was his reaction to Cantor's transfinite numbers, which Kronecker found hard to accept. He may be right: given the set of natural numbers, human intellect has created an assortment of new classes of numbers: negative numbers; rational numbers (fractions); algebraic numbers; irrational numbers; real numbers; transcendental numbers; imaginary numbers and complex numbers (see Appendix 1). These number classes have all found their way into descriptions of physical processes and systems and undoubtedly form the basis of Wigner's (1959) lecture titled the 'Unreasonable effectiveness of mathematics in the natural sciences' and the follow-up publication (Wigner 1960). Because these number classes can be derived from manipulation of the natural numbers, once we understand the ontology and epistemology of the natural numbers, understanding of all other number classes will follow.

The physical world of becoming is an imitation of the mathematical world of being. This view is echoed over the centuries by many mathematicians: Hilbert argued that mathematical truth was independent of the existence of God or other a priori assumptions. Once we study the natural numbers, they seem to assume a life of their own. If we consider the subset of natural numbers $1-10$, the numbers 2 , 3,5 and 7 are prime numbers. ${ }^{7}$ They cannot be divided without remainder by any number found between 1 and the number itself. The numbers 4, 6, 8, 9 and 10 are composite, as these can be written as the product of two or more numbers greater than 1 and less than the composite number. Numbers have properties of their own. The mathematician G.H. Hardy, quoted in Ferguson (1994), stated:

317 is a prime number, not because we think so, or because our minds are shaped in one way or another, but because it is so, because mathematical reality is built that way. (p. 63)

But do the natural numbers exist in reality outside the human imagination? We would like to believe that numbers are universal and recognised by intelligent beings in other galaxies which are too far away to ever be in touch with earthlings. These intelligent beings would in some way or other arrive at the same conclusions as we do when studying those numbers. Perhaps, they are intellectually more advanced and discover properties of the numbers of which we are totally ignorant at present.

But what is a natural number? The ancient Greeks in their study of geometry saw number as a multiple or fraction of a unit length of a line drawn in the sand. The concept of a rational number, which is the ratio of two integers (excluding zero as the denominator), followed naturally from extending or subdividing a given line. But the unit of counting as we see it now is a discrete indivisible object. The collection of countable objects has the property that these objects can be distinguished by some means which allows us to count the objects, that is, exhaustively pair the objects with the set of natural numbers. Surely, God created the environment in which natural numbers can exist, and left it to intelligent beings to discover those properties.

The mathematician and cosmologist Roger Penrose (2004) gives a physics-based example of a discrete countable phenomenon which hints that numbers have physical existence of their own: electric charge in an isolated body always occurs in integral multiples of the charge on the 7.The first prime number is 2 , by definition. If 1 is considered prime, factorisation of composite numbers would not be unique. 
proton or electron. ${ }^{8}$ Electric charge is an instance of what is called an additive quantum number: in order to derive the charge on a composite body, we use standard arithmetic and add up the constituent individual charges, taking into account that some of the constituent charges may be negative.

Wolfram's cellular automaton approach presupposes that space and time ultimately take on a discrete characterisation. The Planck length, named after Max Planck, the originator of quantum theory, is a miniscule length 32 orders of magnitude smaller than $1 \mathrm{~mm}$ and 12 orders of magnitude smaller than the proton (Wikipedia 2017g). Planck time is 44 orders of magnitude smaller than a standard second (Wikipedia 2017h). Quantum theorists suspect that at the Planck length, quantum effects become dominant, and may well break space and time, or Einstein's space time up into discrete chunks. This assumption will support the view that at the most fundamental level, time and space lose their appearance of continuity, and resolve into discrete forms, analogous to the natural number system.

By the various (human) constructions, the ontology of real and complex numbers can be traced back to the ontology of the natural numbers.

\section{Conclusion}

The Pythagorean School viewed the essence of number as the cause of the existence of the universe, and so it remained until Cantor in the late 19th century initiated a deep study to understand the fundamental properties of numbers. He showed that the cardinality of the natural numbers are transfinite and have counter-intuitive properties: the elements of an infinite set may be put into a one-to-one correspondence with the elements of a proper subset of the set, which contradicts Euclid's axiom that the whole is greater than the part. Bertrand Russell and others disclosed that naïve set theory leads to antimonies not compatible with mathematical truth. These developments inspired early 20th century mathematicians to rigorously examine the foundations of mathematics. The leading figure in this venture was the German mathematician David Hilbert, who strove to find a set of axioms that would provide a firm basis in proving postulates and theorems in mathematics. He was surprised when Kurt Gödel published a proof that such a set of axioms cannot exist. Gödel disclosed in his incompleteness theorems that there are statements about numbers that are true but cannot be proven in the number system itself. The philosopher Unger characterised this development as that mathematics was driven from its paradise of undeniable truth and has not been able to return since then. The

8.The empirical observation that the magnitudes of the electric charges on the electron and positron are exactly equal is a cosmic mystery. Since the charges are of opposite sign, a configuration with the same number of protons and electrons is electrically neutral.

9.This smallest unit charge has recently been subdivided by scientists into smaller units. The family of new fundamental particles, the quarks, has fractional charges. The up quark has a positive charge of $2 / 3$; the down quark has a negative charges. The up quark has a positive charge of $2 / 3$; the down quark, has a negative charge of giving i giving it a net charge of +1 . The neutron is a configuration consisting of two down quarks and one up quark, giving it a net charge of zero. The electron, which is fundamental particle, has a charge of -1 . incompleteness theorems of Gödel were extended by the theoretical computer scientist Alan Turing who introduced the notion of computable numbers. These are, in present-day idiom, mathematical functions that can be computed in a finite number of steps on a digital computer.

New possibilities in constructing models of the universe in which we live were opened up by the emergence of the digital computer. The computer scientist Stephen Wolfram proposed that the universe is digital in nature, and runs on fundamental laws which can be characterised by simple programmes akin to cellular automata. Edward Fredkin, Seth Lloyd and Jürgen Schmidhuber have developed the branch of philosophy known as digital philosophy which supports digital models of the universe with various properties. The physicist and philosopher Max Tegmark made the bold assertion that 'all structures which exists mathematically also exist physically'. Tegmark and others have hypothesised that nature is based on well-behaved Turing-complete (computable) functions that will allow humans to eventually comprehend the fundamental structure of the universe. Tegmark's theory is considered to be a form of monism in that it assumes that only mathematical objects exist in the universe.

Many mathematicians, physicists and computer scientists view reality as ultimately a reflection of the postulated digital nature of the universe. Penrose presents the example of electric charge that supports the notion that discrete number entities exist in the physical universe. This is reinforced by the quantum-mechanical view that at the deepest level, time and space are discrete, losing their appearance of continuity. Discreteness implies digital properties, which can be traced back through the rational, real and complex number systems to the natural numbers. If we fully understand the ontology and epistemology of the natural numbers, it will assist in understanding the reality of the cosmos.

These ideas are hypotheses in need of further observations, insights and revelations to prove their value in the ongoing debate on the nature of reality. Until then, nature and reality in the final analysis remain a mystery to us.

\section{Acknowledgements Competing interests}

The author declares that he has no financial or personal relationships which may have inappropriately influenced him in writing this article.

\section{References}

Ball, W. 1960, A short account of the history of mathematics, 4th edn., Dover Publications, New York, pp. 50-62. (Reprint. Original publication: London: Macmillan \& Co., 1908).

Barrow, J., 2000, The Universe that discovered itself, Oxford University Press, Oxford, p. 276.

Bell, E., 1986, Men of mathematics, Simon and Schuster, New York, p. 477

Chaitin, G., 2007, Metamaths: The quest for Omega, Paperback edn., Atlantic Books, London.

Dauben, J., 1977, 'Georg Cantor and Pope Leo XIII: Mathematics, theology, and the infinite', Journal of the History of Ideas 38(1), 85-108. https://doi. org/10.2307/2708842 
Dauben, J., 1979, 'Georg Cantor and Pope Leo XIII: Mathematics, theology, and the infinite', Journal of the History of Ideas 38(1), 85-108, 120, 143. https://doi. org/10.2307/2708842

Ferguson, K., 1994, The fire in the equations: Science, religion and the search for God, Bantam Press, London, p. 63.

Gleiser, M., 2014, The Island of knowledge: The limits of science in the search for meaning, Chapter 12, Basic Books, Perseus Books Group, New York.

Gödel, K., 1931, ‘Über formal unentscheidbare Sätze der Principia Mathematica und verwandter Systeme, I' [On formally undecidable propositions of Principia Mathematica and related systems I], Monatshefte für Mathematik und Physik 38, 173-198. https://doi.org/10.1007/BF01700692

Hilbert, D., 1926, 'Über das Unendliche', Mathematische Annalen 95, 161-190. https://doi.org/10.1007/BF01206605

Lloyd, S., 2006, Programming the universe: A quantum computer scientist takes on the Cosmos, Knopf Doubleday Publishing Group, New York.

Penrose, R., 1989, The Emperor's New Mind: Concerning computers, minds, and the laws of physics, Oxford University Press, Oxford.

Penrose, R., 2004, The road to reality: A complete guide to the laws of the universe, Vintage Books, London.

Schrecker, P \& Schrecker, A. (eds.), 1965, Monadology and other philosophical essays, Prentice-Hall, Upper Saddle River, NJ.

Stewart, I., 1996, From here to infinity, Oxford University Press, Oxford, p. 65.

Tegmark, M., 2014, Our mathematical universe: My quest for the ultimate nature of reality, Penguin Books, Random House, UK, London.

Turing, A., 1937, 'On computable numbers, with an application to the entscheidungsproblem', Proceedings of the London Mathematical Society 2(42), 230-265. https://doi.org/10.1112/plms/s2-42.1.230

Turing, A., 1938, 'On computable numbers, with an application to the entscheidungsproblem: A correction', Proceedings of the London Mathematical Society 2(43), 544-546. https://doi.org/10.1112/plms/s2-43.6.544
Unger, R. \& Smolin, L., 2015, The singular universe and the reality of time: A proposal in natural philosophy, Cambridge University Press, Cambridge.

Wigner, E., 1959, 'The unreasonable effectiveness of mathematics in the natural sciences. Richard Courant lecture in mathematical sciences delivered at New York University, May 11, 1959', Communications on Pure and Applied Mathematics 13 1-14. https://doi.org/10.1002/cpa.3160130102

Wigner, E., 1960, 'The unreasonable effectiveness of mathematics in the natura sciences', Communications on Pure and Applied Mathematics 13, 1-14. https:// doi.org/10.1002/cpa.3160130102

Wikipedia, 2017a, Euclidean geometry, Wikimedia Foundation, Inc., edited on 1 July 2017, viewed 12 July 2017, from https://en.wikipedia.org/wiki/Euclidean geometry

Wikipedia 2017b, Georg Cantor, Wikimedia Foundation, Inc., edited on 09 July 2017, viewed 10 July 2017, from https://en.wikipedia.org/wiki/George Cantor

Wikipedia, 2017c, Gottfried Wilhelm Leibniz, Wikimedia Foundation, Inc., edited on 12 July 2017, viewed 12 July 2017, from https://en.wikipedia.org/wiki/Gottfried Wilhelm_Leibniz

Wikipedia, 2017d, Goldbach's Conjecture, Wikimedia Foundation, Inc., edited on 15 June 2017, viewed 10 July 2017, from https://en.wikipedia.org/wiki/Goldbach's Conjecture

Wikipedia, 2017e, Gregory Chaitin, Wikimedia Foundation, Inc., edited on 09 April 2017, viewed 10 July 2017, from https://en.wikipedia.org/wiki/Greory_Chaitin

Wikipedia, 2017f, Konrad Zuse, Wikimedia Foundation, Inc., edited on 01 July 2017 viewed 10 July 2017, from https://en.wikipedia.org/wiki/Konrad_Zuse

Wikipedia, 2017g, Planck length, Wikimedia Foundation, Inc., edited on 01 July 2017, viewed 10 July 2017, from https://en.wikipedia.org/wiki/Planck_length

Wikipedia, 2017h, Planck time, Wikimedia Foundation, Inc., edited on 02 July 2017 viewed 10 July 2017, from https://en.wikipedia.org/wiki/Planck_time

Wikipedia, 2017i, Pythagoras, Wikimedia Foundation, Inc., edited on 05 July 2017 , viewed 10 July 2017, from https://en.wikipedia.org/wiki/Pythagoras

Wolfram, S., 2002, A new kind of science, Wolfram Media Inc., Champaign, IL. 


\section{Appendix 1}

The Romans did not use a symbol for zero in their subtractive numeral system. The word nulla meaning 'nothing' was used to indicate zero. The famous Italian mathematician Fibonacci (c 11751250 ) introduced the Hindu-Arabic numeral system in Europe in which ' 0 ' denoted both the number zero (the additive identity) and a place holder in the place-value notation of numbers. The negative integers are related to the natural numbers through the solution of the equation $x+n=0$, that is, $x=-n$.

The ancient Greeks used rational numbers, being the ratio of two natural numbers $m / n$, where $n$ may not be zero. An algebraic number is the solution of a polynomial in an unknown $x$ with rational number coefficients, for example, $x^{2}-\frac{3}{2} x+\frac{1}{2}=0$. It is easy to check that the equation has two solutions, namely $x=\frac{1}{2}$ and $x=1$. The equation $x^{2}-2=0$ has the solution $x$ is equal to the square root of 2 . The Pythagoreans, circa 2500 years ago, discovered that the square root of 2 cannot be expressed as a rational number $m / n$ for any two whole numbers $m$ and $n$. These numbers are referred to as surds or irrational numbers.

Irrational numbers are a subset of the real numbers. There exist real numbers that are not the solution of any algebraic equation. Real numbers were defined by in the late 18th century by the Dedekind cut, named after the mathematician Richard Dedekind, who devised a method to intersperse the real numbers into the rational numbers, which, as presented above, are derived from the natural numbers.

The equation $x^{2}+1=0$, that is, $x^{2}=-1$, has no solution in the real numbers. There is no number, positive or negative, that when multiplied with itself yields a negative number. Mathematicians postulated an imaginary number denoted by $i$ with the property that $i^{2}=-1$. Complex numbers are a combination of real and imaginary numbers, such as $3+4 i$. These numbers are of vital importance in mathematical descriptions of wave propagation, particularly electromagnetic waves and their connection with quantum theory. 